\title{
Avaliação da superfície dos instrumentos ProTaper Next e Logic após diferente número de usos, através da Microscopia Eletrônica de Varredura
}

Surface evaluation of ProTaper Next and Logic after different use numbers, throughout scanning electron microscopy

Evaluación de la superficie de los instrumentos ProTaper Next y Logic después de diferentes usos, utilizando microscopía electrónica de barrido

Received: 03/30/2021 | Reviewed: 04/09/2021 | Accept: 04/11/2021 | Published: 04/21/2021

Paulo Eduardo Silva Santos ORCID: https://orcid.org/0000-0001-8580-5841 Faculdade São Leopoldo Mandic, Brasil E-mail: pauloeduardozz@hotmail.com

Ana Grasiela da Silva Limoeiro ORCID: https://orcid.org/0000-0003-4633-720X CESUPI - Faculdade de Ilhéus, Brasil E-mail: grasielalimoeiro@gmail.com

Wayne Martins Nascimento ORCID: https://orcid.org/0000-0003-4201-4710 Faculdade São Leopoldo Mandic, Brasil E-mail: waynemartinsn@gmail.com

Carlos Eduardo da Silveira Bueno ORCID: https://orcid.org/0000-0002-2675-0884 Faculdade São Leopoldo Mandic, Brasil E-mail: carlosesbueno@terra.com.br

Cesar Augusto Perini Rosas ORCID: https://orcid.org/0000-0002-2234-5531 Universidade Estadual do Norte do Paraná, Brasil E-mail: cesarperini66@hotmail.com Augusto Shoji Kato ORCID: https://orcid.org/0000-0003-2971-0906 Universidade de São Paulo, Brasil E-mail: endo.kato@gmail.com

\begin{abstract}
Resumo
A reutilização das limas endodônticas é uma prática comum entre os profissionais, embora seu uso único seja recomendado pelo fabricante. O presente estudo teve como objetivo comparar a superfície das limas de dois sistemas rotatórios fabricados em NiTi tratadas termicamente: Limas \#25/01 e \#25/06 do sistema ProDesign Logic e as limas X1 e X2 do sistema ProTaper Next, após 4 etapas: sem uso, primeiro, segundo e terceiro uso, através da análise sob microscopia eletrônica de varredura. Foram utilizados no total de 60 incisivos inferiores com apenas um canal e 20 conjuntos de limas, divididos em 4 grupos $(n=10)$, de acordo com os diferentes tempos de uso. As limas do grupo controle foram submetidas a esterilização e posicionadas no aparelho de Microscopia Eletrônica de Varredura e as imagens das superfícies foram obtidas e serviram como imagem controle. Na sequência, as limas foram submetidas a primeira instrumentação nos dentes extraídos, lavadas em cuba ultrassônica com detergente enzimático e novamente analisadas no MEV. Repetiu-se esses procedimentos até o terceiro uso. Utilizou-se da estatística qualitativa, para a presença ou não da alteração de superfície, e aplicação do teste qui-quadrado de partição (p<0.05). Os resultados demonstraram que não houve diferença significante entre a presença de alterações nos tempos experimentais, sendo que depois do primeiro uso, todas as limas $(25.01,25.06, \mathrm{X} 1$ e X2) apresentaram alterações na superfície. Conclui-se que, os instrumentos endodônticos utilizados na presente pesquisa sofreram alterações superficiais significativas após o primeiro uso, contraindicando a reutilização dos mesmos.
\end{abstract}

Palavras-chave: Endodontia; Instrumentos odontológicos; Microscopia eletrônica de varredura.

\begin{abstract}
The reuse of endodontic files is a common practice among professionals, although its single use is recommended by the manufacturer. This study aimed to compare the files surface of two heat treated rotating systems manufactured in NiTi: Files \# 25/01 and \# 25/06 of the ProDesign Logic system and files X1 and X2 of the ProTaper Next system, after 4 steps: unused, first, second and third use, through analysis under scanning electron microscopy. A total of 60 lower incisors were used with only one canal and 20 sets of files, divided into 4 groups $(n=10)$, according to the different
\end{abstract}


times of use. The files of the control group were submitted to sterilization and placed in the Scanning Electron Microscopy device and the images of the surfaces were obtained and served as a control image. Subsequently, the files were subjected to the first instrumentation in the extracted teeth, washed in an ultrasonic vat with enzymatic detergent and again analyzed in SEM. These procedures were repeated until the third use. Qualitative statistics were used for the presence or absence of surface alteration and application of the chi-square test of partition $(\mathrm{p}<0.05)$. The results showed that there was no significant difference between the presence of changes in the experimental times, and after the first use, all files $(25.01,25.06, \mathrm{X} 1$ and X2) showed changes on the surface. It is concluded that the endodontic instruments used in the present research underwent significant superficial changes after the first use, contraindicating their reuse.

Keywords: Endodontics; Dental instruments; Scanning electron microscopy.

\section{Resumen}

La reutilización de limas endodónticas es una práctica habitual entre los profesionales, aunque el fabricante recomienda su uso único. El presente estudio tuvo como objetivo comparar la superficie de los archivos de dos sistemas rotativos tratados térmicamente fabricados en NiTi: los archivos \# 25/01 y \# 25/06 del sistema ProDesign Logic y los archivos X1 y X2 del sistema ProTaper Next, después de 4 pasos: sin usar, primer, segundo y tercer uso, mediante análisis bajo microscopía electrónica de barrido. Se utilizaron un total de 60 incisivos inferiores con un solo canal y 20 juegos de limas, divididos en 4 grupos $(n=10)$, según los diferentes tiempos de uso. Los archivos del grupo de control se sometieron a esterilización y se colocaron en el dispositivo de microscopía electrónica de barrido y las imágenes de las superficies se obtuvieron y sirvieron como imagen de control. Posteriormente, las limas fueron sometidas a la primera instrumentación sobre los dientes extraídos, lavadas en tina ultrasónica con detergente enzimático y nuevamente analizadas en el SEM. Estos procedimientos se repitieron hasta el tercer uso. Se utilizó estadística cualitativa para la presencia o ausencia de cambio de superficie y la aplicación de la prueba de partición de chi-cuadrado (p <0.05). Los resultados mostraron que no hubo diferencia significativa entre la presencia de cambios en los tiempos experimentales, y después del primer uso, todas las limas (25.01, 25.06, X1 y X2) mostraron cambios en la superficie. Se concluye que los instrumentos de endodoncia utilizados en la presente investigación sufrieron cambios superficiales significativos después del primer uso, contraindicando su reutilización.

Palabras clave: Endodoncia; Instrumentos dentales; Microscopía electrónica de barrido.

\section{Introduçãa}

A efetiva limpeza e modelagem do sistema de canais radiculares determina os objetivos biológicos e mecânicos do tratamento endodôntico (Schilder, 1974). O preparo do canal radicular em canais atrésicos com curvaturas severas, quando realizado com limas de aço inoxidável pode causar a formação de desvios, degraus e perfurações (Pecora \& Capelli, 2006). Com objetivo de minimizar a ocorrência desses acidentes, as limas de níquel titânio (NiTi) tratadas termicamente, foram desenvolvidas, agregando benefícios às propriedades, tais como efeito de memória de forma e superelasticidade (Walia et al., 1988), fase martensítica e R, fio Mwire e memória controlada (CM), tornando-as mais resistentes à fadiga cíclica (Peixoto et al., 2010) e menos susceptíveis a desvios em canais curvos quando comparadas aos instrumentos de aço inoxidável (Celik et al., 2013).

Independente do sistema utilizado, múltiplos ciclos de esterilização afetam diretamente a eficiência de corte das limas rotatórias de NiTi (Rapisarda et al., 1999), fazendo com que a limpeza do canal seja insatisfatória, trazendo sérios riscos para o tratamento endodôntico. Diferentes metodologias podem ser aplicadas para avaliar o desgaste das limas endodônticas de NiTi, sendo elas: perfilométrica (Yguel-Henry et al., 1990), a perda de peso (Campos et al., 2021; Vinothkumar et al. 2007), a microtomografia computadorizada (Shen et al., 2008) e também a análise da superfície através da microscopia eletrónica de varredura (MEV). O sistema ProDesign Logic (Bassi/Easy, Belo Horizonte, Brasil), produzido em liga de NiTi com tratamento térmico CM, segue o conceito de lima única após patência enquanto as limas ProTaper Next (Dentsply Maillefer, Suiça) são produzidas em liga de NiTi com o tratamento térmico MWire, e apresenta 3 limas principais (X1, X2 e X3) e mais 2 limas opcionais (X4 e X5).

O objetivo deste estudo foi avaliar através da Microscopia Eletrônica de Varredura (MEV) o estado físico dos sistemas ProDesign Logic e ProTaper Next depois da sua primeira utilização. A hipótese nula é de que não exista diferença entre os sistemas avaliados em diferentes momentos de uso. 


\section{Metodologia}

Foram selecionados 60 incisivos centrais e laterais inferiores humanos, apresentando apenas um único canal, reto e ápices completamente formados, obtidos do Biorepositório da Faculdade São Leopoldo Mandic (Parecer do comitê de ética número 2.115.855). Todos os dentes foram radiografados com aparelho de RX PROCION Ion - 70X e sensor digital Microimagem, nos sentidos vestíbulo-lingual e mesio-distal para verificação da anatomia interna e presença ou ausência de obstruções. Foram Incluídos neste estudo, os dentes que não possuíam tratamento endodôntico, que não apresentavam nenhum tipo de obstrução no conduto radicular e comprimento real do dente maior ou igual a $20 \mathrm{~mm}$. Foram excluídos dentes tratados endodonticamente, com calcificação total ou parcial dos canais radiculares, apresentando trincas e fraturas, forame apical com maior diâmetro que a lima \#15 e com rizogênese incompleta. Com o objetivo de padronizar o acesso coronário, foi utilizada uma ponta diamantada esférica \#1012. Após a exposição da câmara pulpar, as interferências coronárias foram removidas com broca diamantada 3071, promovendo acesso direto ao canal. Uma lima tipo K-File \#10 foi introduzida no canal 1,0 mm além do forame e em seguida, uma lima tipo K-File \#15 foi posicionada no forame apical. Os dentes com forame apical de diâmetro maior que o correspondente a lima \#15 foram descartados. Um stop de silicone foi posicionado na borda incisal, determinando o comprimento real do dente. Para essa medida, quando necessário, desgastes na borda incisal foram realizados com disco de aço, obtendo-se o comprimento real de $20 \mathrm{~mm}$. Dessa medida, $1 \mathrm{~mm}$ foi subtraído, determinando o comprimento de trabalho (CT) em $19 \mathrm{~mm}$. Todos os procedimentos anteriormente descritos foram realizados com auxílio de microscópio operatório com magnificação de 8 vezes (Aliance, São Carlos, São Paulo). O número de espécimes foi baseado em cálculo amostral tendo como referência os resultados dos testes pilotos. Foram determinados dois grupos experimentais e analisados três momentos distintos para cada um.

Para o momento 0 (grupo controle) os instrumentos ProDesign Logic sem uso foram esterilizados em autoclave. As limas ProTaper Next já vêm esterilizadas pela fábrica, assim, foram montados em um stub com auxílio de uma pinça, presa a uma fita adesiva de carbono e submetido a análise de superfície em MEV com aumento de 200X. As imagens obtidas foram arquivadas em formato digital em um pendrive e transferidas para o software Adobe Photoshop.

Para o momento 1 (grupo PL1: n=10) uma lima tipo K-File \#15 foi introduzida em cada canal radicular até o CT. O canal foi irrigado com solução de $\mathrm{NaOCl}$ a $2.5 \%$ com volume de $15 \mathrm{~mL}$ para cada dente, utilizando uma seringa descartável de $5 \mathrm{~mL}$ e agulha Navitip (30 ga - 0,33 $\mathrm{mm}$ ) a $2 \mathrm{~mm}$ do CT. A lima 25.06 foi acionada em $950 \mathrm{rpm}$ e torque $4 \mathrm{~N}$ para realizar a instrumentação do terço cervical e médio com movimento de vai e vem. A cada manobra com amplitude de 3 movimentos, a lima era retirada, limpa com uma gaze, o dente irrigado com solução de $\mathrm{NaOCl}$, o líquido aspirado com um sugador endodôntico e a lima introduzida novamente até atingir o comprimento provisório de trabalho ( $2 \mathrm{~mm}$ aquém do CT). Após essa manobra, foi realizado uma nova irrigação e introdução de uma lima \#10 até o forame. Foi realizada a irrigação e aspiração do líquido, e a lima 25.01 foi acionada com velocidade de $350 \mathrm{rpm}$ e torque $1 \mathrm{~N}$ com movimento de vai e vem até o forame, foi realizada a irrigação e utilizado novamente a lima 25.06 como instrumento final até o CT. Em seguida as limas foram lavadas em cuba ultrasônica, secas em papel absorvente e levadas para o MEV sempre com o auxílio de uma pinça e obtidas as imagens dos primeiros $5 \mathrm{~mm}$ do instrumento e na medida de $10 \mathrm{~mm}$ da ponta. As imagens obtidas foram arquivadas em formato digital e transferidas para o software Adobe PhotoShop. Em seguida, as limas foram para uma cuba ultra-sônica contendo detergente enzimático e água quente por 10 minutos para realização da limpeza. Todas as limas foram padronizadas com um sulco referencial, feito com um disco de carburundum no cabo do instrumento, para observar o número de vezes de utilização.

Para o momento 2 (PL2) e momento 3 (PL3) os procedimentos foram idênticos do momento 1 . O momento 1 referente ao grupo PN (PN1), seguiu o protocolo semelhante ao grupo PL1, apenas modificando o torque (2N) e a velocidade (300 RPM) conforme indica o fabricante. Para o momento 2 (PN2) e momento 3 (PN3) os procedimentos foram idênticos do momento 1. 
Após a obtenção de todas as imagens, foram realizadas as análises e verificação das possíveis alterações encontradas. O software Bioestat 5.0 foi utilizado para análise dos dados e estabelecido o nível a $=5 \%$.

\section{Resultados}

Foram observadas as seguintes alterações: Micro-trinca e desgaste do ângulo de corte das limas (Figura 1).

Inicialmente analisou-se da ponta da lima até $5 \mathrm{~mm}$ verificando após cada uso a presença ou não da mudança na superfície da lima. Para determinar as diferenças significativas foi aplicado os resíduos do qui-quadrado, sendo o valor 4.1404 maior que os valores de referência $1.96(0.05)$ e $2.576(0.01)$, portanto diferença altamente significante $(\mathrm{p}<0.0001)$ para as limas Logic \#25.06 e X2 ProTaper Next que apresentaram significativamente menor número de mudança de superfície no primeiro uso. Houve tendência crescente e significativa com os usos sucessivos no aparecimento de alterações sendo que após o primeiro uso todas as limas apresentaram mudança na superfície (Tabela 1).

Figura 1. Imagens em MEV das limas ProDesign Logic e ProTaper Next, antes e após o primeiro uso. (A, B) - ProDesign Logic 25.01, (C, D) - ProDesign Logic 25.06, (E, F) - ProTaper Next X1, (G, H) - ProTaper Next X2. (A, C, E, G) - Sem uso. (B, D, F, G) - Após primeiro uso.

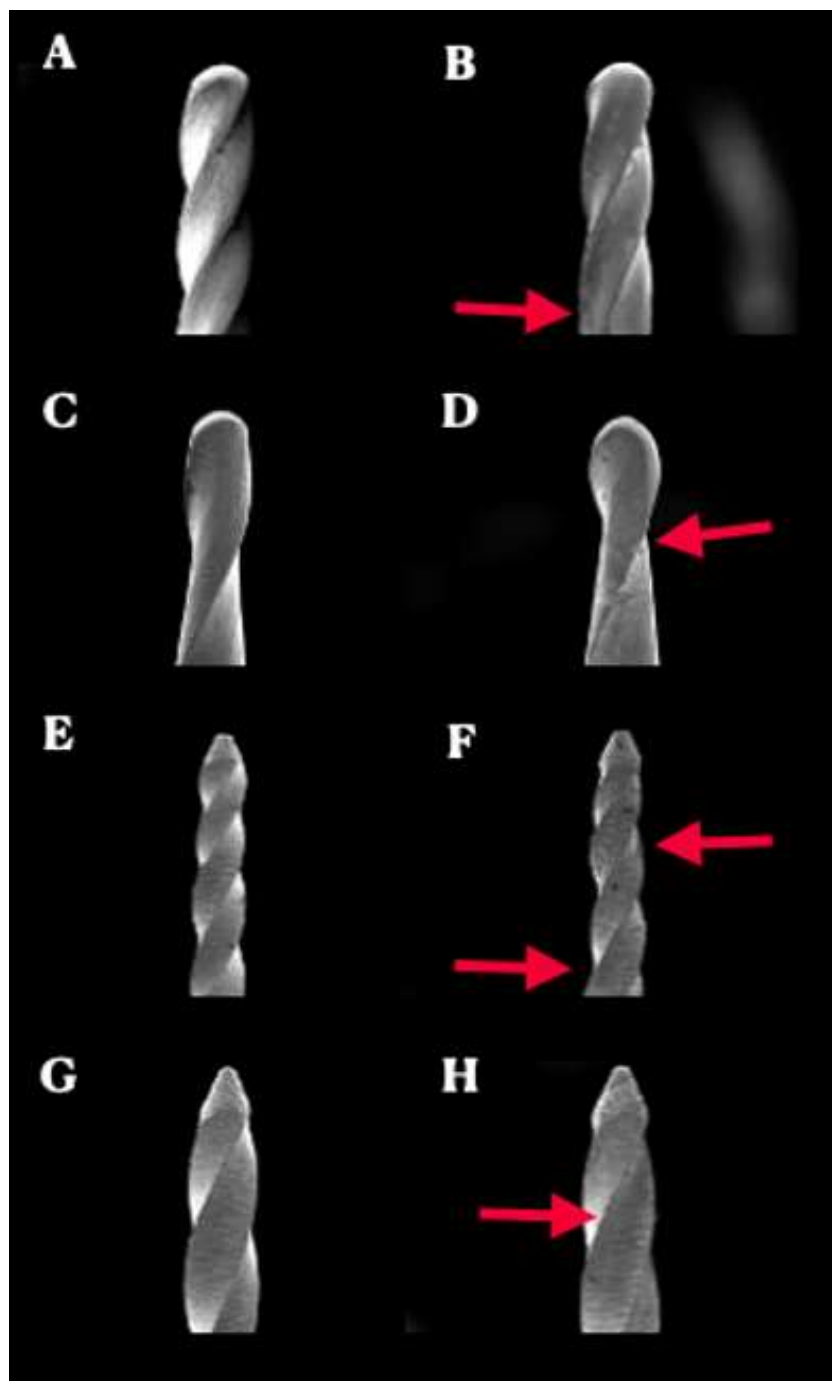

Fonte: Autores. 
Tabela 1. Presença ou ausência de defeitos da ponta da lima até $5 \mathrm{~mm}$, sem uso e após sucessivos usos.

\begin{tabular}{|c|c|c|c|c|c|c|c|c|}
\hline $\begin{array}{c}\text { Ponta até } \\
5 \mathrm{~mm}\end{array}$ & $\begin{array}{l}\text { PDL } \\
25.01\end{array}$ & $\begin{array}{l}\text { PDL } \\
25.01\end{array}$ & $\begin{array}{l}\text { PDL } \\
25.06\end{array}$ & $\begin{array}{l}\text { PDL } \\
25.06\end{array}$ & $\begin{array}{l}\text { PN } \\
\text { X1 }\end{array}$ & $\begin{array}{l}\text { PN } \\
\text { X1 }\end{array}$ & $\begin{array}{l}\text { PN } \\
\text { X2 } \\
\end{array}$ & $\begin{array}{l}\text { PN } \\
\mathbf{X} 2 \\
\end{array}$ \\
\hline & SIM & NÃO & SIM & NÃO & SIM & NÃO & SIM & NÃO \\
\hline SEM USO & 0 & 10 & 0 & 10 & 0 & 10 & 0 & 10 \\
\hline PRIMEIRO & 4 & 6 & 2 & 8 & 5 & 5 & 2 & 8 \\
\hline SEGUNDO & 10 & 0 & 10 & 0 & 10 & 0 & 10 & 0 \\
\hline TERCEIRO & 10 & 0 & 10 & 0 & 10 & 0 & 10 & 0 \\
\hline
\end{tabular}

* PDL - ProDesign Logic, PN - ProTaper Next. SIM - Presença de defeitos. NÃO - Ausência de defeitos. Fonte: Autores.

Foram analisadas também as imagens, $10 \mathrm{~mm}$ aquém da ponta. Para determinar as diferenças significativas foi aplicado os resíduos do qui-quadrado, sendo o valor 4.4113 maior que os valores de referência 1.96 (0.05) e 2.576 (0.01), portanto diferença altamente significante $(\mathrm{p}<0.0001)$ para as limas Logic \#25/06 e X2 ProTaper Next que apresentaram significativamente menor número de alterações de superfície no primeiro uso. Houve tendência crescente com os usos sucessivos do aparecimento de alterações (Tabela 2).

Tabela 2. Presença ou ausência de defeitos a $10 \mathrm{~mm}$ da ponta, sem uso e após sucessivos usos.

\begin{tabular}{|c|c|c|c|c|c|c|c|c|}
\hline $10 \mathrm{~mm}$ & $\begin{array}{c}\text { PDL } \\
25.01\end{array}$ & $\begin{array}{c}\text { PDL } \\
25.01\end{array}$ & $\begin{array}{l}\text { PDL } \\
25.06\end{array}$ & $\begin{array}{c}\text { PDL } \\
25.06\end{array}$ & $\begin{array}{l}\text { PN } \\
\text { X1 }\end{array}$ & $\begin{array}{l}\text { PN } \\
\text { X1 }\end{array}$ & $\begin{array}{l}\text { PN } \\
\text { X2 }\end{array}$ & $\begin{array}{l}\text { PN } \\
\text { X2 }\end{array}$ \\
\hline & SIM & NÃO & SIM & NÃO & SIM & NÃO & SIM & NÃO \\
\hline SEM USO & 0 & 10 & 0 & 10 & 0 & 10 & 0 & 10 \\
\hline PRIMEIRO & 3 & 7 & 1 & 9 & 5 & 5 & 1 & 9 \\
\hline SEGUNDO & 10 & 0 & 10 & 0 & 10 & 0 & 10 & 0 \\
\hline TERCEIRO & 10 & 0 & 10 & 0 & 10 & 0 & 10 & 0 \\
\hline
\end{tabular}

* PDL - ProDesign Logic, PN - ProTaper Next. SIM - Presença de defeitos. NÃO - Ausência de defeitos. Fonte: Autores.

\section{Discussão}

O presente estudo analisou a superfície de dois sistemas rotatórios (ProTaper Next e ProDesign Logic) em diferentes tempos de instrumentação. Foram observadas mudanças na superfície em ambas as limas, como micro trincas e desgaste do ângulo de corte após o primeiro uso, portanto, a hipótese nula foi rejeitada. Essas mudanças na superfície ocorreram em todas as limas após o primeiro uso, agravando-se após o segundo e terceiro uso. Não houve fratura de nenhuma lima durante o experimento.

Inan et al., 2009, utilizando limas de NiTi rotatórias MTwo, mostraram que a fadiga cíclica é a principal causa das fraturas dos instrumentos e ressaltaram a importância de não exceder o número máximo de uso recomendado pelo fabricante. Devido as alterações físicas dos instrumentos, a importância de utilizar limas de NiTi apenas uma vez para evitar o risco de fratura deve ser avaliada (Arens et al., 2003; Shen et al., 2009). A resistência de um instrumento à fratura fica comprometida após o uso clínico repetido (Vieira et al., 2008), mesmo que não haja nenhum sinal visivelmente detectável de defeitos (Barbosa et al., 2007).

A presença de curvaturas impõe dificuldades nos procedimentos de limpeza e modelagem (Miranzi et al., 2011), porém as limas devem resistir aos procedimentos de dilatação e manter o canal centralizado sem fraturar. Nesse sentido vários tratamentos térmicos foram propostos para a superfície das limas de NiTi, que resultaram em maior resistência ao desgaste do que as limas de NiTi convencional (Alapati et al., 2011; Shen et al.,2011; Shen et al., 2012; Jia \& Yong 2012). Entretanto, as limas de NiTi que possuem tratamento térmico não determinaram melhoras significativas quando comparadas às limas convencionais de $\mathrm{NiTi}$, apresentando micro trincas e outras alterações nas suas superfícies (Tsujimoto et al., 2014). 
Vários ciclos de uso clínico e esterilização das limas rotatórias Hyflex, não apresentaram diminuição na eficiência de corte e flexibilidade com 1 uso, mas perda da flexibilidade com 2 usos (Seago et al. 2015). Esses achados vão ao encontro dos resultados da presente pesquisa que evidenciou que após o primeiro uso a presença de deformações foi nítida e que a esterilização não determinou alterações ou trincas. O número de ciclos de autoclave não desempenhou um papel significativo na capacidade dos instrumentos Hyflex CM ou ProFile ISO de manter a curvatura original do canal (Thompson et al., 2013).

Esta pesquisa não foi conclusiva sobre a determinação do número de uso possível para os sistemas ProTaper Next e ProDesign Logic. O tratamento térmico da liga do instrumento ProDesign Logic e sua seção transversal parecem influenciar a resistência à fadiga cíclica (de Menezes et al., 2017). No preparo do canal radicular, o termo "lima única" refere-se à utilização de um único instrumento, porém os instrumentos podem ser usados em três vezes devido ao uso em dentes multirradiculares na prática clínica. A esterilização e a reutilização desses tipos de instrumentos não são recomendadas devido a potenciais deficiências de desempenho (Coelho et al., 2016).

Limas com os menores diâmetros de ponta devem ser consideradas como instrumentos descartáveis pela maior possibilidade de fadiga torcional (Fernández-Pazos et al., 2018; Shen et al. ,2009 b). A utilização dos instrumentos por mais vezes, além da questão da fratura do instrumento, deve-se levar em consideração, também, a alteração superficial do instrumento, pois este está intimamente relacionada com a capacidade de limpeza e modelagem do canal radicular. Para reduzir o risco de fratura dos instrumentos dentro do canal, todas as limas devem ser examinadas após cada uso, e mesmo antes do seu primeiro uso, pois defeitos de fabricação podem causar a fratura de um novo instrumento (Fernández-Pazos et al., 2018).

\section{Conclusão}

Através dos resultados obtidos neste estudo, concluiu-se que, os instrumentos endodônticos utilizados na presente pesquisa sofreram alterações superficiais significativas após o primeiro uso, contra-indicando a reutilização dos mesmos. É necessário que o clínico leve em consideração todas as variáveis presentes durante o uso clínico ao determinar a capacidade de um instrumento ser reutilizado para instrumentação subsequente.

\section{Referências}

Alapati, S. B., Brantley, W. A., Iijima, M., Clark, W. A., Kovarik, L., Buie, C., \& Johnson, W. B. (2009). Metallurgical characterization of a new nickel-titanium wire for rotary endodontic instruments. Journal of endodontics, 35(11), 1589-1593.

Arens, F. C., Hoen, M. M., Steiman, H. R., \& Dietz Jr, G. C. (2003). Evaluation of single-use rotary nickel-titanium instruments. Journal of Endodontics, 29(10), 664-666.

Barbosa, F. O. G., Gomes, J. A. D. C. P., \& de Araújo, M. C. P. (2007). Influence of previous angular deformation on flexural fatigue resistance of K3 nickeltitanium rotary instruments. Journal of endodontics, 33(12), 1477-1480.

Campos, A. E. A., de Jesus Soares, A., da Silva Limoeiro, A. G., Cintra, F. T., Frozoni, M., \& Campos, G. R. (2021). Cutting efficiency of ProDesign R, Reciproc Blue and WaveOne Gold reciprocating instruments. Research, Society and development, 10(3), e1710313028-e1710313028.

Çelik, D., Taşdemir, T., \& Er, K. (2013). Comparative study of 6 rotary nickel-titanium systems and hand instrumentation for root canal preparation in severely curved root canals of extracted teeth. Journal of endodontics, 39(2), 278-282.

Coelho, B. S., Amaral, R. O. J. F. D., Leonardi, D. P., Marques-da-Silva, B., Silva-Sousa, Y. T. C., Carvalho, F. M. A. D., \& Baratto-Filho, F. (2016). Performance of three single instrument systems in the preparation of long oval canals. Brazilian dental journal, 27(2), $217-222$.

Da Cunha Peixoto, I. F., Pereira, É. S. J., da Silva, J. G., Viana, A. C. D., Buono, V. T. L., \& de Azevedo Bahia, M. G. (2010). Flexural fatigue and torsional resistance of ProFile GT and ProFile GT series X instruments. Journal of endodontics, 36(4), 741-744.

De Menezes, S. E. A. C., Batista, S. M., Lira, J. O. P., \& de Melo Monteiro, G. Q. (2017). Cyclic fatigue resistance of WaveOne Gold, ProDesign R and ProDesign Logic files in curved canals in vitro. Iranian endodontic journal, 12(4), 468.

Fernández-Pazos, G., Martín-Biedma, B., Varela-Patiño, P., Ruíz-Piñón, M., \& Castelo-Baz, P. (2018). Fracture and deformation of ProTaper Next instruments after clinical use. Journal of clinical and experimental dentistry, 10(11), e1091. 
Inan, U., \& Gonulol, N. (2009). Deformation and fracture of Mtwo rotary nickel-titanium instruments after clinical use. Journal of endodontics, 35(10), 13961399.

Miranzi, B. A. S., Borges, G. A., Miranzi, A. J. S., Menezes, F. H. D., Miranzi, M. A. S., \& Borges, L. H. (2011). Apical and cervical displacement produced by hand and engine-driven stainless steel and nickel-titanium instruments in simulated curved root canal. Brazilian Journal of Oral Sciences, 10(2), 136-139.

Pécora, J. D., \& Capelli, A. (2006). Shock of paradigms on the instrumentation of curved root canals. Brazilian dental journal, 17(1), 3-5.

Rapisardaa, E., Bonaccorsob, A., Tripib, T. R., \& Condorellic, G. G. (1999). Effect of sterilization on the cutting efficiency of rotary nickel-titanium endodontic files. Oral Surgery, Oral Medicine, Oral Pathology, Oral Radiology, and Endodontology, 88(3), 343-347.

Schilder H. (1974). Cleaning and shaping the root canal. Dent Clin North Am, 18:269-296.

Seago, S. T., Bergeron, B. E., Kirkpatrick, T. C., Roberts, M. D., Roberts, H. W., Himel, V. T., \& Sabey, K. A. (2015). Effect of repeated simulated clinical use and sterilization on the cutting efficiency and flexibility of Hyflex CM nickel-titanium rotary files. Journal of endodontics, 41(5), 725-728.

Shen, Y., Coil, J. M., \& Haapasalo, M. (2009a). Defects in nickel-titanium instruments after clinical use. Part 3: a 4-year retrospective study from an undergraduate clinic. Journal of Endodontics, 35(2), 193-196.

Shen, Y., Coil, J. M., Mclean, A. G., Hemerling, D. L., \& Haapasalo, M. (2009b). Defects in nickel-titanium instruments after clinical use. Part 5: single use from endodontic specialty practices. Journal of endodontics, 35(10), 1363-1367.

Shen, Y., Qian, W., Abtin, H., Gao, Y., \& Haapasalo, M. (2012). Effect of environment on fatigue failure of controlled memory wire nickel-titanium rotary instruments. Journal of endodontics, 38(3), 376-380.

Shen, Y., Qian, W., Abtin, H., Gao, Y., \& Haapasalo, M. (2011). Fatigue testing of controlled memory wire nickel-titanium rotary instruments. Journal of endodontics, 37(7), 997-1001.

Shen, Y., \& Haapasalo, M. (2008). Three-dimensional analysis of cutting behavior of nickel-titanium rotary instruments by microcomputed tomography. Journal of endodontics, 34(5), 606-610.

Thompson, M., Sidow, S. J., Lindsey, K., Chuang, A., \& McPherson III, J. C. (2014). Evaluation of a new filing system's ability to maintain canal morphology. Journal of endodontics, 40(6), 867-870.

Tsujimoto, M., Irifune, Y., Tsujimoto, Y., Yamada, S., Watanabe, I., \& Hayashi, Y. (2014). Comparison of conventional and new-generation nickel-titanium files in regard to their physical properties. Journal of endodontics, 40(11), 1824-1829.

Vieira, E. P., França, E. C., Martins, R. C., Buono, V. T. L., \& Bahia, M. G. A. (2008). Influence of multiple clinical use on fatigue resistance of ProTaper rotary nickel-titanium instruments. International Endodontic Journal, 41(2), 163-172.

Vinothkumar, T. S., Miglani, R., \& Lakshminarayananan, L. (2007). Influence of deep dry cryogenic treatment on cutting efficiency and wear resistance of nickel-titanium rotary endodontic instruments. Journal of endodontics, 33(11), 1355-1358.

Walia, H., Brantley, W. A., \& Gerstein, H. (1988). An initial investigation of the bending and torsional properties of Nitinol root canal files. Journal of endodontics, 14(7), 346-351.

Ye, J., \& Gao, Y. (2012). Metallurgical characterization of M-Wire nickel-titanium shape memory alloy used for endodontic rotary instruments during lowcycle fatigue. Journal of endodontics, 38(1), 105-107.

Yguel-Henry, S., Vannesson, H., \& Von Stebut, J. (1990). High precision simulated cutting efficiency measurement of endodontic root canal instruments: influence of file configuration and lubrication. Journal of endodontics, 16(9), 418-422. 